(C) 2016 IEEE. Personal use of this material is permitted. Permission from IEEE must be obtained for all other uses, in any current or future media, including reprinting/republishing this material for advertising or promotional purposes, creating new collective works, for resale or redistribution to servers or lists, or reuse of any copyrighted component of this work in other works.

The published version can be found at: http://dx.doi.org/10.1109/ICC.2016.7511315 


\title{
Multipath Routing Approach to Enhance Resiliency and Scalability in Ad-hoc Networks
}

\author{
Alexandros Ladas, Nikolaos Pavlatos, Nuwan Weerasinghe and Christos Politis \\ Wireless Multimedia \& Networking (WMN) Research Group \\ Faculty of Science, Engineering and Computing (SEC) \\ Kingston University London, KT1 2EE London, United Kingdom \\ Email: \{A.Ladas, k1444618, Nuwan.Weerasinghe, C.Politis\}@kingston.ac.uk
}

\begin{abstract}
This paper presents Multipath-ChaMeLeon (MCML) as an update of the existing ChaMeLeon (CML) routing protocol. CML is a hybrid and adaptive protocol designed for Mobile Ad-Hoc Networks (MANETs), supporting emergency communications. M-CML adopts the attributes of the proactive Optimized Link State Protocol (OLSR) and extends it so as to implement a multipath routing approach based on the Expected Transmission Count (ETX). The paper substantiates the efficiency of the protocol through a simulation scenario within a MANET using the NS-3 simulator. The acquired results indicate that M-CML routing approach combined with an intelligent link metric such as the ETX reduces the effects of link instabilities and enhances the network performance in terms of resiliency and scalability.

Keywords-MANETs, Multipath routing, ETX
\end{abstract}

\section{INTRODUCTION}

Routing protocol optimization for Mobile Ad hoc Networks (MANETs) has emerged as a growing field of research in the area of wireless communication technologies. MANETs are self-configuring, decentralized wireless network models, composed by a number of mobile nodes operating in a distributed mode, without the use of an administrator or an access point. Their autonomous topology allows the creation of non-permanent, purpose-built networks that can perform under dense conditions. MANETs are considered as an excellent solution to accommodate emergency situation scenarios, such as rescue or disaster relief operations, with the view to provide reliable communication and contribute on the protection of human lives.

However, the dynamic topology in which ad hoc networks typically operate increases the complexity of the protocol design. Nodes may join or leave the network instantaneously, while the restricted energy resources may result to abrupt disconnections. Link instabilities, produced by external factors such as propagation delay or interference, negatively impact the bit error rate, generating failures. Within this context, the challenge that arises brings forward an optimization problem, where the routing protocol needs to formulate strategies for satisfying quality of service $(\mathrm{QoS})$ requirements, while being able to adapt to the radical topology changes and maintain satisfying energy consumptions.

Unlike the typical routing protocols of centralized networks which take advantage of the Dynamic Host Control Protocol (DHCP) as a fixed IP addressing scheme, Ad Hoc routing protocols require decentralized and multi-hop mechanisms. Each node must be able to create paths and undertake routing decisions while collaborating with the rest of the network. In this light, ad hoc routing protocols are classified in two categories based on their implemented routing discovery mechanism. Reactive routing protocols such as Ad-hoc On-demand Distance Vector (AODV) [1] or Dynamic Source Routing (DSR) [2], support bandwidth conservation by establishing ondemand end-to-end routes. They remain in a sleep mode until a data transmission occurs, which triggers the route detection process. Conversely, proactive routing protocols such as OSLR [3], allow the periodic exchange of topology information, offering constant route discovery and maintenance. Their table driven functionality is based in the constant distribution of control messages among the network's nodes, as a mechanism to identify the network's topology and instantaneously forward data.

The different characteristics of proactive and reactive approaches emerge a fundamental trade-off. On the one hand side, the proactive dissemination reduces the latency of transmission, provides readily available information, but increases the generating routing overhead. On the other hand side, reactive routing discovery approach reduces the generation of redundant routing messages, classifying it as a better candidate for operating within large networks, but suffers from the increased delay of data delivery.

In this paper, we provide an update on the adaptive and hybrid CML routing protocol [4] [5] [6] [7] which adapts its routing behavior according to changes in the network size. For small networks, CML routes data proacticely using the OLSR routing protocol, and utilizes the reactive AODV protocol while operating in large networks. The paper proposes a multipath approach of CML called M-CML, implemented on the proactive phase of CML. The reactive phase is considered dormant at this stage. M-CML is based on OLSR routing protocol, extended so as to calculate multiple routing paths based on the Expected Transmission Count (ETX) [8] instead of the traditional hop count. ETX is an alternative link metric, which calculates the quality of the link based on the number of successful transmissions. It aims to provide increased resiliency of performance and reduce the probability of link errors. In addition to the ETX metric, M-CML abolishes the single path allocation used by the original OLSR, and implements 
a multipath routing approach. Mobility, network's scalability and other environmental factors, influence the sensitive nature of MANETs, making nodes and links prone to errors and failures. The multipath approach aims to enhance the reliable communication by simultaneously transmit data to all possible routes.

\section{RELATED WORK}

The design of the proactive phase of CML adopts the OLSR characteristics which relies on its capability to constantly update topology information. Nodes flood the network with link state information messages consisted by the periodic transmissions of HELLO and TC messages, which perform the route discovery and maintenance process. Through the exploitation of the received information nodes manage to determine the most optimal path to the destination based on the minimum number of hops. HELLO messages are generated and transmitted exclusively to 1-hop neighbors and are not any further relayed to the rest of the nodes of the network. Their main task is to sense the link quality and allocate the set of 1-hop and 2-hop neighbors, and identify the willingness of a node to act as a Multipoint Relays (MPRs). The elected MPRs compose a distinctive flooding mechanism responsible to generate TC messages, with the view to identify the rest of the network's topology and store it within the topology information base. Each node within the network maintains and updates its routing table in order to forward data to the destination based on minimum hops.

Hop count is utilized as the default link metric of OLSR designated for its simplicity to adapt and be implemented, where each router within a constructed path receives information regarding the next hop. Hop count is not based on the distance of the sender and the receiver but on the minimum hops required to reach the destination so as to abbreviate the end-to-end delay and increase throughput. However, attributes of major importance, such as packet loss, reliability, latency and bandwidth are not evaluated. It has been shown that a route that minimizes the hop count does not necessarily maximize a flow's throughput [9]. Nevertheless, hop count metric has been used by the most significant Ad hoc routing protocols like OLSR and AODV as the default link metric.

The constantly growing popularity of ETX has attracted the attention of the research community. Its implementation in DSDV routing protocol by Zaidi et al. in [10] indicate that when alternative paths have similar ETX value summary, selecting such a link quality metric is insignificant and results to low throughput proportions. On the other hand side, De Couto et al. in [8] investigate the functionality of DSDV by comparing the ETX with the hop count metric and indicate that there is a negative impact on the size of the routing overhead, but it is considered insignificant compared to the sufficient increase of throughput. Finally Liu et al. in [11] performs the same comparison in OLSRv2. The authors recognized that ETX performs better when the paths are longer and the traffic is higher but is flawed when paths are small and traffic is considered light. The contradiction in terms of performance between ETX and hop count makes the comparison decision an open question till these days. There are many different factors that influence the performance of the two metrics such as the particular routing protocol in which the metric is incorporated, as well as the simulation environment.

Route discovery is considered the most important factor to determine the willingness of the protocol to adopt a reactive or a proactive approach. However, the constant effort to enhance the MANET's performance has emerged another important criterion which is the ability of a protocol to allocate and transmit information through a single or multiple paths. Multipath routing protocols have distracted the attention of MANET research community which has implemented and examined a variety of routing approaches and implementations towards this field. Authors in [12] propose an OLSR solution based on multipath calculations in terms of bandwidth and delay to improve the QoS. The protocol through the use of the shortestwidest path algorithm manages to compute disjoint and loop free paths. On the other hand, Yi et al. through [13], take into consideration the node's reliability based on multipath routing to enhance the TCP data transmission. Experimental evaluation concluded that the multipath approach offers higher throughput and connection resilience compared to the single path version. Finally authors in [14] came to the conclusion that multipath reactive routing protocols increase the number of RREQ messages during execution, resulting additional overhead while intermediate nodes become more vulnerable to duplicate packets.

\section{TheORETICAL BACKGROUND}

This section presents the fundamental concepts on which MCML lays its foundation. It introduces the protocol's theoretical axes by discussing the multipath approach as an efficient routing solution to improve the transmission reliability and outlines the theoretical foundation of the ETX link metric.

\section{A. Multipath Routing}

Single path protocols are susceptible to link and node failures confronting instabilities during execution due to the network's mobility and energy restrictions. The multipath approach has been proposed for Ad hoc routing protocols as an efficient solution to address the challenges of scalability, mobility and link instability of the network. Nodes maintain a routing table which comprises all network destinations, while the periodic exchange of control messages offers efficient route discovery without any extra overhead or energy costs. This classifies the OLSR protocol as a suitable candidate to interact with the multipath attributes. The synchronous data transmission through multiple paths aims to accommodate the challenges that arise in constantly changing environments and improve the reliability of the communication. However, the multipath approach confronts challenges such as the generation of duplicated packets and bandwidth utilization. In addition, the frequently changing topology increases the probability of generated loops while transmitting data through 
intermediate nodes and increases the necessity of designing route recovery techniques.

\section{B. Expected Transmission Count (ETX)}

The unsatisfactory network performance of hop count has emerge the need to identify and propose more intelligent link metric solutions. Within this scope, M-CML considers ETX as the metric to measure the link quality by calculating the estimated number of transmissions required for a node to successful transmit a single packet towards that link. As

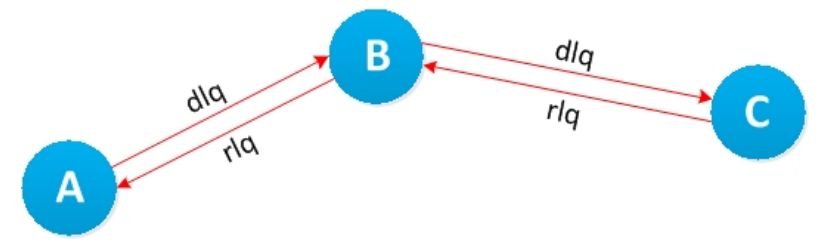

Fig. 1. ETX functionality

illustrated in Fig. 1, loss rates are computed in both directions. Node A to B calculates the direct link quality $(d l q)$ as the probability of a successful transmission within a window period. Similarly, Node B to A calculates the probability of a successful transmission on the reception $(r l q)$. The ETX metric concept can easily be implemented through the periodic exchange of HELLO messages which allocate the 1-hop and 2-hop neighbors within a certain time interval.

$$
E T X=\frac{1}{d l q * r l q}
$$

Based on (1) the quality of the link is considered perfect, when the $\mathrm{ETX}=1$, whereas the number of HELLO messages attached to the receiver are equal with the total HELLO messages transmitted by the sender. The use of HELLO messages simplifies the link quality evaluation of the path compared to the use of data packets. Node A while acting as a receiver is not aware of the number of packets that node B wants to transmit. However, it is able to estimate the total number of HELLO messages based on Node's B HELLO time interval.

\section{SySTEM MODEL}

This section presents the implemented methodology for the design of M-CML. In this paper we modify the ETX computed formula so as to conform with the software features, in line with the specifications of [15]:

$$
E T X=d l q * r l q
$$

Unlike hop count's objective which is to allocate a route using the least possible hops, ETX aims to identify and calculate a path with the minimum ETX summary. M-CML implements an advanced relay routing mechanism which allows it to reduce the improvident generation of duplicated packets. Nodes within a MANET maintain and update their routing table by comprising a set of available next hop addresses and the corresponding ETX values sorted in an ascending order, based on the minimum ETX values. Instead of flooding the network with redundant information, the transmitted data is forwarded restrictly to the paths that carry the two most optimal ETX values (minimum ETX values). The following subsections firstly introduce the modifications applied to the proactive phase of CML in order to calculate paths based on the ETX link metric, and secondly describe the adopted methodology used for the multipath routing.

\section{A. Message format}

The proposed protocol adopts the HELLO message periodic exchange mechanism for populating the neighborhood and local link information base and extends its content so as to include the $r l q$ and ETX values of the link. Each node within a MANET stores a set of link tuples named link set to represent the topology information of the network with information obtained through the periodic exchange of HELLO messages. Link tuples are an important mechanism for the ETX calculation as part of the link sensing process and are modified for the purposes of the M-CML so as to encompass the values of $d l q$, $r l q$ and ETX. Similar to HELLO messages, TC messages are updated so as to include a vector of ETX values, corresponding to the advertised neighbor addresses encompassed within TC messages. TC messages use the information acquired during the link sensing and neighbor detection processes in order to disseminate topology information throughout the network as part of the protocol's flooding process.

\section{B. Message processing}

As previously explained, the periodic exchange of HELLO messages among 1-hop neighbors facilitates the implementation of the ETX calculation within the link sensing process. Link sensing is responsible for the maintenance of the node's link information base which stores information about the node's association with its neighbors. This way link tuples can efficiently store information about the ETX attributes to the link. On receipt of a HELLO message, the local link information base is initially checked to identify whether an association to the sender of the HELLO message already exists. If no link tuple is attached to the local link information base, then a new tuple with the following characteristics pertaining to the ETX metric is created:

- Number of HELLO received $=1$

- Number of HELLO supposed $=1$

- $r l q=1$

- $d l q=\mathrm{UNDEFINED}=0$

- last packet sequence number

- diff: the difference between the current packet sequence number and the last sequence number

- $E T X=$ DEFAULT METRIC VALUE = 4096

- ETX SEQNO RESTART DETECTION = 256

In the possibility that the link tuple already exists within the local link information base, the information gets updated according to the link messages carried by the received HELLO messages. In particular, the link message has to assess whether the HELLO message link code is valid or invalid based on the 
probability of a lossy link. Each time the link is valid (not lost), the local link information base gets updated with the new link tuple only if the corresponding ETX value is more optimal compared to the existing one. During the ETX calculation, the link's asymmetry is not taken into consideration and the $d l q$ is considered equal to $r l q$ that the message carries.

Similar to the link set, the 2-hop Neighbor Set is accordingly updated through the periodic exchange of HELLO messages which allow the maintenance of a symmetric link to a symmetric neighbor [3]. Each time a new HELLO message is acquired the 2-hop Neighbor Set updates its 2-hop tuples including the ETX values carried by the link messages. Essentially, the ETX information carried by the 2-hop neighbor measures the information between the link quality of the 1-hop and the 2hop neighbor.

The TC message processing involves the maintenance and constant update of the topology information of the network through the use of the topology set. The topology set is updated each time a new TC message is received following the example of the Link Set and the 2-hop Neighbor Set. In particular, in case the topology tuple is not included within the topology set, then the topology set creates a new tuple with the corresponding ETX value. If the tuple is already part of the topology set it has to be updated with the new ETX value carried by the advertised neighbour main address of the TC message. TC messages offer nodes the ability to obtain the ETX value corresponding to the generator of the message and its own MPR selectors. TC messages are carrying the ETX value so as to offer link information to the topology set. The ETX value of the TC message essentially evaluates the quality of the link between the MPR selector and the message generator. On receipt of a TC message, the message is scanned, the ETX values carried are copied and stored to the corresponding topology tuple.

\section{Multipath Routing}

Each entry in the routing table corresponds to a destination. Nodes within a MANET create and maintain a routing table which holds information about the routes to the destination addresses. M-CML classifies the routing table entries based on number of hops enforcing the re-computation of the routing table following the principles of the proactive phase of CML whenever a change occurs in the link set, neighbor set, 2-hop neighbor set and topology set based on the following concept:

- For all the 1 hop neighbors the ETX value recorded within the routing table is equal to the ETX metric of the link tuple.

- For all the 2 hop neighbors, the ETX value recorded within the routing table is equal to the most optimal ETX value corresponding to the available one hop neighbor, plus the ETX value of the 2-hop neighbor tuple connected to this 1-hop neighbor.

- The rest of the network's nodes are being accommodated by the topology tuples. Hence the ETX metric is equal to the ETX value obtained by the topology tuple, plus the
ETX value of the neighbor associated with that topology tuple.

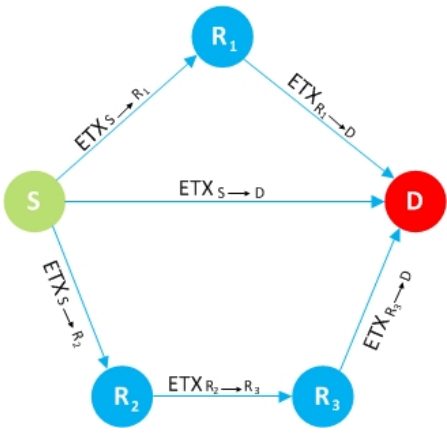

Fig. 2. Overview of M-CML approach

M-CML's routing table entries allows the storage of multiple paths for each recorded destination in the network. Each destination within the routing table comprise a set of next addresses. This set of addresses correspond to the available next hop nodes, representing the $N$ available paths. Each next address of the set of next addresses is followed by the corresponding ETX values, sorted in ascending order starting from the minimum ETX value i.e. (nextaddr1, ETX1), (nextaddr2, ETX2),..., (nextaddrn, ETXn, where ETX1 <ETX2<..<ETXn. This way the routing table provides readily available information of the mutliple paths per destination.

To reduce the generation of multiple duplicated messages produced due to the multipath concept, M-CML implements a simplified approach by sending data to the two most optimal disjoint next addresses based on the information extracted by the ETX value. The proposed routing protocol extends the Route Output process of the RFC 3626 by designing a gateway list. Its process is triggered whenever data is to be transmitted towards a destination. In particular, the gateway list allocates the routing entry corresponding to the requested destination, parses the already sorted in an ascending order ETX values and transmits the data based on the two minimum ETX values.

\section{EVAluation}

\section{A. Simulation Setup}

In the light of this paper, a simulation scenario is implemented using NS3 simulator [16] to facilitate the project experimentation purposes and the collection of data for the evaluation of the M-CML performance. The paper's experimental setup, is implemented within a $800 \times 800$ meter area using the ns3::RandomRectanglePositionAllocator class, as a mean to install the simulation nodes randomly within the investigating area. The nodes' transmission power is set to $7.5 \mathrm{dBm}$, while the ns3::RandomWaypointMobilityModel class is used as a suitable mobility standard able to assign random waypoints on each node. This allows the network's nodes to move randomly based on the proposed speed and pause time. The traffic type used is CBR applicable for voice and audio applications with low latency, whereas the physical layer modulation technique used for the purposes of this project is OFDM with a rate of 
6Mbps using the IEEE 802.11a standard for wireless networks. Results are acquired by increasing the number of nodes while the number of pairs (source/destinations) remains constant. A summary of the simulation parameters is presented in the table below.

TABLE I

SimULATION SET

\begin{tabular}{|l||l|}
\hline \multicolumn{2}{|c|}{ Network Parameters } \\
\hline Network topology & $800 \mathrm{~m} \times 800 \mathrm{~m}$ \\
\hline Simulation Time & 350 seconds \\
\hline Start up Time & 50 seconds \\
\hline Node Speed & $6 \mathrm{~m} / \mathrm{sec}$ \\
\hline Number of nodes & $8,10,12,14,16,18,20,22$ \\
\hline Source-Sinks data pairs & 3 \\
\hline Packet Size & 256 bytes \\
\hline
\end{tabular}

\section{B. Simulation Results}

In this subsection we evaluate the performance of M-CML and OLSR in terms of:

- Packet delivery ratio (PDR): The proportion of successful data packets delivered to the destination compared to the total generated data packets.

- Average end-to-end delay: The mean time required for the surviving data packet to traverse the distance from the source to the destination.

- Normalized routing load: The sum of the transmitting control messages divided by the sum of the delivered data in bytes.

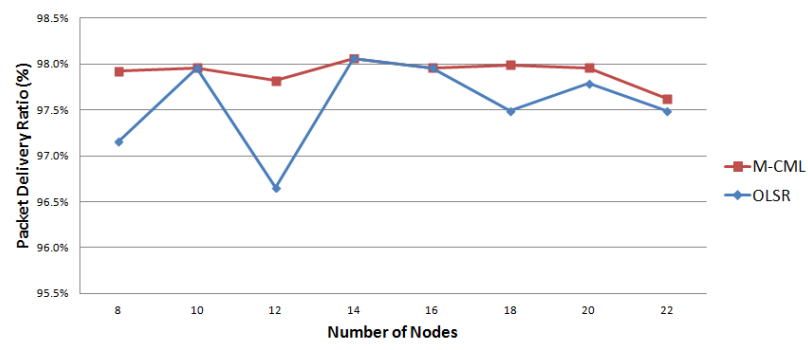

Fig. 3. Packet delivery ratio against network size

The performance of M-CML in terms of PDR as illustrated in Fig. 3 indicates a more robust performance throughout the simulation. The OLSR's single path performance instabilities while operating with 8 and 12 nodes can be interpreted as a consequence of using the ns3::randomrectanglepositionallocator. Initially, nodes are randomly installed within the investigating area, which in the case of this scenario negatively impacts the path availability resulting to link failures and errors. On the other hand side, M-CML efficiently confronts the node random distribution and addresses the challenges that occur due to lossy links based on its multipath feature. However the increase of node density indicates a leverage for OLSR while operating with 22 nodes. MCML attribute to allocate and transmit data through more than one paths results to the generation of duplicates packets that impacts the protocol's performance. Attributes like bandwidth utilization, energy consumption affect the protocol's efficiency as the network's load increases.

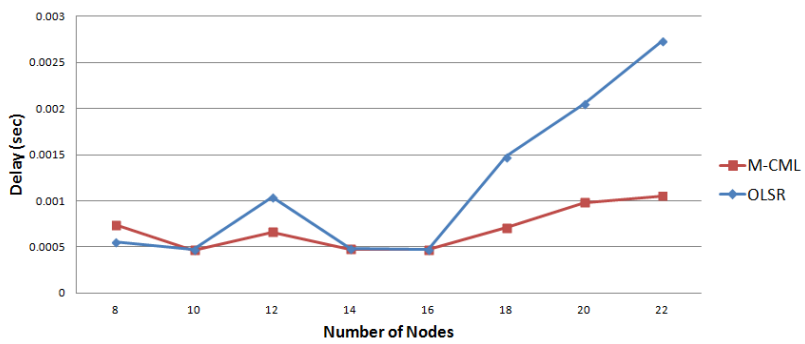

Fig. 4. End-to-end delay against network size

There is a relevant behaviour in terms of end-to-end delay when operating between 8 to 16 nodes, as presented in Fig. 4. The effects of the nodes random distribution is once more visible when operating with 12 nodes. As the node density increases M-CML indicates significant resilience compared to OLSR. This can be explained by the fact that OLSR suffers from the propagation delay by forwarding packets through longer paths based on minimum hop count, whereas M-CML transmits packets based on the ETX link metric. In addition, the multiple availability of paths to reach the destination reduces the impact of the queue delay which increases the probability of collisions on the transmission process.

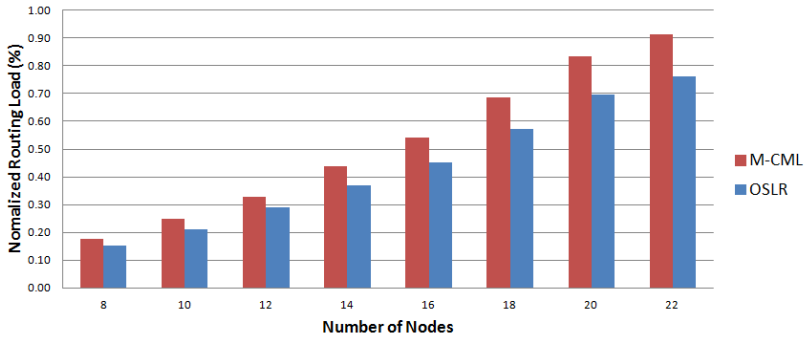

Fig. 5. Normalized routing load against network size

Fig. 5 illustrates a contradiction between the proposed protocol and the original OLSR. The ETX metric increases the size of the routing overhead. By introducing additional information within the control messages, the augmentation of the routing load is inevitable. In addition to the ETX metric, the multipath approach affects the computational costs of the routing table by calculating and maintaining all the available routes, reducing the memory capacity and the network's life span.

\section{CONCLUSion AND Future Work}

This paper investigated the design, implementation and experimentation results of M-CML which extends the proactive phase of CML. The first stage of the design of M-CML involved the ETX calculation at the link sensing stage through the exploitation of the periodic exchange of control messages. The second stage introduced of a routing table computation 
algorithm for the maintenance of the available routes per destination. Finally, the third stage, involved the selection of the two most optimal paths for transmission based on ETX to increase the propability of successful transmission.

Carried out simulations within a MANET, investigated the proposed protocol scalability by increasing the node density while nodes were constantly moving within a fixed area using the NS-3 simulator.

The acquired results introduced a trade-off. On the one hand side, M-CML's multipath routing combined with ETX offered a significant reduction of end-to-end delay compared to OLSR. More packets were able to successfully reach the destination increasing the PDR proportions confining link instabilities and enhancing the resiliency of performance. On the other hand side, the utilization of ETX in the proposed protocol resulted in the need to incorporate additional information within the generated control messages which led to a surge in the normalized routing load. In addition, the multipath approach triggered the generation of redundant messages. By using two paths to send the same information the creation of multiple duplicated messages was inevitable.

Additional considerations around the computational cost pertaining to the calculation of multiple paths and the increase of memory storage for the routing table were discussed and will need to be further investigated as part of future work. In addition the design and implementation of the multipath ETX based routing extension of OLSRv2 will be investigated and compared with our proposed approach.

\section{ACKNOWLEDGMENT}

This work was supported by the European Union's FP7 Project SALUS (Security and Interoperability for the Next Generation of PPDR Communication Systems) 2013 - 2016, Grant agreement no:313296

\section{REFERENCES}

[1] C. Perkins, E. Belding-Royer, and S. Das, "Ad hoc On-Demand Distance Vector (AODV) Routing," RFC 3561 (Experimental), Internet Engineering Task Force, July 2003. [Online]. Available: http://www.ietf.org/rfc/rfc3561.txt

[2] D. Johnson, Y. Hu, and D. Maltz, "The Dynamic Source Routing Protocol (DSR) for Mobile Ad Hoc Networks for IPv4," RFC 4728 (Experimental), Internet Engineering Task Force, February 2007. [Online]. Available: http://www.ietf.org/rfc/rfc4728.txt

[3] T. Clausen and P. Jacquet, "Rfc3626: The optimized link state routing protocol,' The Internet Engineering Task Force, 2003. [Online]. Available: http://www.ietf.org/html.charters/manet-charter.html

[4] A. Ramrekha, E. Panaousis, and C. Politis, "ChaMeLeon (CML): A hybrid and adaptive routing protocol for Emergency Situations," (Experimental), Internet Engineering Task Force, February 2011. [Online]. Available: http://tools.ietf.org/html/draft-ramrekha-manet-cml02

[5] A. Ramrekha and C. Politis, "A hybrid adaptive routing protocol for extreme emergency ad hoc communication," in Computer Communications and Networks (ICCCN), 2010 Proceedings of 19th International Conference on, aug. 2010, pp. 1 -6.

[6] A. Ramrekha, G. Millar, and C. Politis, "A model for designing scalable and efficient adaptive routing approaches in emergency ad hoc communications," in Computers and Communications (ISCC), 2011 IEEE Symposium on, 28 2011-july 1 2011, pp. 916 -923.
[7] A. Ramrekha, O. Adigun, A. Ladas, N. Weerasinghe, and C. Politis, "Towards a scalable routing approach for mobile ad-hoc networks," in 20th IEEE International Workshop on Computer Aided Modelling and Design of Communication Links and Networks, 7-9 September 2015.

[8] D. S. J. De Couto, D. Aguayo, J. Bicket, and R. Morris, "A high-throughput path metric for multi-hop wireless routing," Secaucus, NJ, USA, pp. 419-434, Jul. 2005. [Online]. Available: http://dx.doi.org/10.1007/s11276-005-1766-z

[9] L.-W. Chen, W. Chu, Y.-C. Tseng, and J.-J. Wu, "Route throughput analysis with spectral reuse for multi-rate mobile ad hoc networks," WOS:000269626300017, 2009. [Online]. Available: https://ir.nctu.edu.tw/handle/11536/6772

[10] Z. Zaidit, T. Tan, and Y. Cheng, "Etx could result in lower throughput," in Computer Communications and Networks, 2009. ICCCN 2009. Proceedings of 18th Internatonal Conference on, Aug 2009, pp. 1-6.

[11] N. Liu and W. Seah, "Performance evaluation of routing metrics for community wireless mesh networks," in Intelligent Sensors, Sensor Networks and Information Processing (ISSNIP), 2011 Seventh International Conference on, Dec 2011, pp. 556-561.

[12] H. Badis and K. Al Agha, "Qolsr multi-path routing for mobile ad hoc networks based on multiple metrics: bandwidth and delay," in Vehicular Technology Conference, 2004. VTC 2004-Spring. 2004 IEEE 59th, vol. 4, May 2004, pp. 2181-2184 Vol.4.

[13] J. Yi, E. Cizeron, S. Hamma, and B. Parrein, "Simulation and performance analysis of mp-olsr for mobile ad hoc networks," in Wireless Communications and Networking Conference, 2008. WCNC 2008. IEEE, March 2008, pp. 2235-2240.

[14] J. Yi, A. Adnane, S. David, and B. Parrein, "Multipath optimized link state routing for mobile ad hoc networks," Ad Hoc Netw. vol. 9, no. 1, pp. 28-47, Jan. 2011. [Online]. Available: http://dx.doi.org/10.1016/j.adhoc.2010.04.007

[15] E. B. H.Rogge and A. Kaplan, "Packet Sequence Number based ETX Metric for Mobile Ad Hoc Networks," The Internet Engineering Task Force, Mar. 2010. [Online]. Available: https://tools.ietf.org/html/draftfunkfeuer-manet-olsrv2-etx-01

[16] G. F. Riley and T. R. Henderson, "The $n s-3$ network simulator," in Modeling and Tools for Network Simulation, 2010, pp. 15-34. [Online]. Available: http://dx.doi.org/10.1007/978-3-642-12331-32 